\title{
Tensor-based Analysis of ECG Changes Prior to In-Hospital Cardiac Arrest
}

\author{
Griet Goovaerts ${ }^{1,2}$, Sabine Van $\mathrm{Huffel}^{1,2}$, Xiao $\mathrm{Hu}^{3}$ \\ ${ }^{1}$ Department of Electrical Engineering (ESAT), STADIUS Center for Dynamical Systems, Signal \\ Processing and Data Analytics, KU Leuven, Belgium \\ 2 imec, Leuven, Belgium \\ ${ }^{3}$ Department of Physiological Nursing, UCSF, San Francisco, USA
}

\begin{abstract}
This works presents an analysis in the changes in beat morphology prior to in-hospital cardiac arrest. We have used tensor decomposition methods to extract features from the ECG signal. After preprocessing and $R$ peak detection, a tensor is constructed for each ECG signal by segmenting the signal in individual heartbeats and stacking them in a $3 D$ manner. The result of the tensor decomposition are 3 factor vectors corresponding to each tensor dimension. The temporal vector, representing the standard heartbeat over all leads in the signal, is further processed to calculate 10 different features: 4 features characterizing global changes in beat morphology and 6 detailed features describing changes in timing and amplitude of the waveforms. We analyzed a dataset of 20 patients who experienced a cardiac arrest in the intensive care unit at the end of the recording. For each patient, a stable signal (in the beginning of the recording) and an unstable signal (near the cardiac arrest) were extracted and processed. Statistical analysis of the results in both time windows (e.g. stable and unstable) show significant changes in the values of 2 out of 4 global parameters and 4 out of 6 detailed parameters. The results indicate that the use of tensor-based methods can be a robust way to characterize ECG changes, and may be a useful tool in identifying patients at risk for cardiac arrest.
\end{abstract}

\section{Introduction}

In-hospital cardiac arrests account for approximately $40 \%$ of all cardiac arrests in the United States. When a patient experiences a cardiorespiratory arrest, a 'code blue' is called, indicating a medical emergency or a person in need of immediate medical attention. Since only $25 \%$ of code blue patients survives to discharge [1], early identification of patients at risk can be crucial to improve patient outcome.

One strategy is to continuously monitor vital signs in pa- tients and identify patterns or changes in these signals that are predictive for a later code blue in that patient. Since ECG is already monitored in many patients, ECG morphology changes are a good candidate for such analysis. There have been many studies investigating the immediate mechanisms that lead to cardiorespiratory arrest, but the preceding changes in the ECG signal on a longer time scale are less well-studied. Previous studies focused on bradyasystolic cardiac arrests [2] or made use of manual measurements [3], which is a time-consuming task when dealing with long-term recordings.

In this work we use tensor-based analysis methods, which have been shown to give robust results in the detection of $\mathrm{T}$ wave alternans [4] and myocardial infarction [5]. They perform a multidimensional analysis which allows simultaneous analysis of different ECG leads.

The next section first gives an overview of the data and methods used in this study. Section 3 presents the results and discussion, followed by a conclusion.

\section{Data and Methods}

\subsection{Data}

We collected a dataset of 20 patients that experienced a code blue due to a cardiopulmonary arrest in the intensive care unit of the UCSF Medical Center. All data were collected between 2013 and 2015. For all patients information about the date and time of the code, the type of cardiac arrest and whether they survive to discharge is available.

For each patient, ECG signals from the last 24 hours before the code blue occurred were extracted from their medical file. All ECG signals were sampled with a sampling frequency of $240 \mathrm{~Hz}$ and contain 7 standard ECG channels: I, II, III, V, aVR, aVL and aVF. We selected two 1-hour periods from each recording: the first hour of the recording (where the patient is presumed to be stable) and the last hour of the recording right before the code blue. These periods will from now on be referred to as respectively the stable signal or period and unstable signal or period. 


\subsection{Preprocessing}

Since data collected in an intensive care unit typically contains a fair amount of noise, preprocessing was necessary to improve the signal quality. Baseline wander and power line interference are therefore removed with respectively a Chebyshev filter with cut-off frequency $0.5 \mathrm{~Hz}$ and a notch filter. After that, $\mathrm{R}$ peaks are automatically detected using Pan-Tompkins [6]. Annotations with outliers in R peak amplitudes (caused by technical artifacts or movement artifacts) that form large spikes in the signal) were removed prior to further analysis. A subset of the $\mathrm{R}$ peak locations were visually inspected to ensure that the detection accuracy was sufficient.

\subsection{Tensor construction}

All signals are processed with a non-overlapping moving window of 100 heartbeats. We will construct, decompose and analyze a tensor for each window, which allows to capture the dynamic changes in the ECG signal over time. 100 heartbeats corresponds to a window length of 0.5-1.5 minutes, depending on the heart rate of the patient. Since the focus of this study is the analysis of changes in heartbeat morphology, we construct a tensor that consists of all heartbeats in the analysis window. The ECG signal is therefore segmented in individual heartbeats and each beat is stacked in a frontal slice of the tensor. The ECG leads form the second (spatial) dimension, the temporal profile of each heartbeat the third dimension. Segmentation length is determined for each window based on the mean RR interval $(\overline{R R})$ in that window. The segmentation window is symmetrical around the $\mathrm{R}$ peak and the window length is $1.6 \overline{R R}$. Note that the segmentation window is rather long, meaning that parts of the previous and/or next heartbeat might be included in the segmentation. This is however not a problem since we will detect the beginning and end of each heartbeat in a later step.

The result is one tensor $\mathcal{T}$ for each analysis window. Each tensor has 3 dimensions: the first dimension is the heartbeats dimension with a fixed length of 100. The spatial dimension correspond to the different ECG leads in the signals and is here equal to 7 . The temporal dimension shows the time course of the individual heartbeat and has a varying length for each tensor, depending on the current heart rate.

\subsection{Tensor decomposition}

We decompose the constructed tensor $\mathcal{T}$ using Canonical Polyadic Decomposition (CPD). While this is considered the most simple tensor decomposition method, it also leads to the most interpretable results. Since the goal is to analyze parameters that can be easily conveyed to medical doctors and patients, interpretability of the results is an absolute necessity. CPD will decompose a tensor in a (minimal) sum of rank-1 terms. The number of rank-1 terms is called the rank of the decomposition. Since we are interested in the major changes in heartbeat morphology, the decomposition rank is set to 1 .

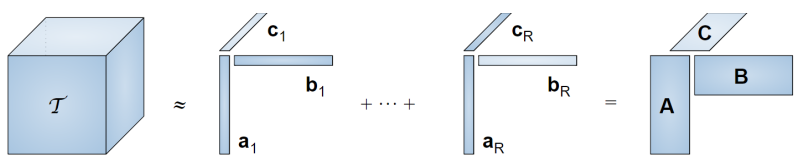

Figure 1: Canonical Polyadic Decomposition [7]

Figure 1 shows a visual representation of this decomposition on a 3D tensor. Note that $\mathrm{R}$ is 1 in our case, so only one component consisting of 3 factor vector $\mathbf{a}_{1}, \mathbf{b}_{\mathbf{1}}$ and $\mathbf{c}_{\mathbf{1}}$ is calculated. The three factor vectors correspond to the three dimensions of the original tensor: time, space and heartbeats.

\subsection{Feature extraction}

The output of the tensor decomposition are 3 factor vectors, one corresponding to each dimension (or mode) of the tensor. The mode- 1 vector shows the distribution over the different ECG channels. The mode- 2 vector of the temporal dimension can be seen as a template heartbeat for all heartbeats included in the tensor. The mode- 3 factor vector shows the differences among all heartbeats in a window. Since the analysis focuses on the changes in ECG morphology, the mode- 2 factor vector will be used for further analysis.

Since the mode- 2 factor vector corresponds to the aggregate of all heartbeats in the analysis window, a standard ECG delineator can be used to automatically detect the peaks of the individual ECG waves. Here we opted for the wavelet-based delineator developed by Martinez et al. [8]. Only the peak of the $\mathrm{P}$ and $\mathrm{T}$ wave are detected since the begin and end points of these waves are often hard to distinguish. All annotations were inspected visually and adjusted where necessary. 10 different features are derived from these annotations: 4 global features that characterize the changes in beat morphology and 6 detailed features that describe the changes in timing and amplitude of the waveforms. For each feature in each window we calculate the relative difference compared to a baseline value, e.g. the value of the feature in the first analysis window.

\subsubsection{Global features}

Dynamic time warping (DTW) is used to examine the changes in the global heartbeat morphology. DTW is a technique to find an optimal alignment between two time 
segments in a non-linear way. A warping path that optimally matches one segment with the other is iteratively determined, and the length of this warping path can be used as a similarity measure: the shorter the warping path, the more similar the segments are. Here, DTW is used to measure the similarity between (part of) the baseline heartbeat (e.g. the first mode- 2 vector of the stable period) and (part of) the mode- 2 factor vectors of each analysis windows. We examine the DTW distance for the complete heartbeat and each ECG wave individually, so in total 4 different time warping features are calculated:

1. DTW $\mathrm{W}_{\text {heartbeat }}$

2. $\mathrm{DTW}_{\mathrm{P}}$

3. $\mathrm{DTW}_{\mathrm{QRS}}$

4. $\mathrm{DTW}_{\mathrm{T}}$

\subsubsection{Detailed features}

The detailed features represent 6 standard time and amplitude measures that are commonly used in ECG analysis. Note that since all signals are normalized in the preprocessing stage, the final three parameters do not present the ECG signal in $\mathrm{mV}$, but rather the normalized amplitudes. This facilitates comparison among subjects. The detailed features are:

1. $\mathrm{PR}$ interval in $\mathrm{ms}(P R)$

2. QRS interval in $\mathrm{ms}(Q R S)$

3. QT interval in ms $(Q T c)$, corrected for heart rate using Fridericia's method [9]:

$$
Q T c=\frac{Q T}{\sqrt[3]{R R}}
$$

4. Amplitude of the $\mathrm{P}$ wave $(p A m p)$

5. Amplitude of the $\mathrm{R}$ wave $(r A m p)$

6. Amplitude of the T wave (tAmp)

\subsection{Statistical analysis}

The differences between the feature values of the stable and the unstable periods are tested with a two-sample t-test $(\mathrm{p}=0.01)$. Additionally, Cohen's $d$ is used to calculate an estimate of the effect size:

$$
\mathrm{d}=\frac{\mu_{\text {stable }}-\mu_{\text {unstable }}}{\sqrt{\left.\sigma_{\text {stable }}^{2}+\sigma_{\text {unstable }}^{2}\right) / 2}}
$$

with $\mu$ and $\sigma$ respectively the mean and standard deviation of the stable and unstable period. Effect sizes can be divided in four groups according to their $d$-values: small (d $>0.2)$, medium $(\mathrm{d}>0.5)$, large $(\mathrm{d}>0.8)$ and very large $(\mathrm{d}>1.3)[10]$.

\begin{tabular}{|l|c|c|}
\hline Global features & p-value & Cohen's d \\
\hline DTW $_{\text {heartbeat }}$ & 0.0004 & 1.0379 \\
DTW $_{P}$ & 0.0156 & 0.7501 \\
DTW $_{\text {QRS }}$ & 0.0017 & 0.9475 \\
DTW $_{T}$ & 0.0376 & 0.6517 \\
\hline Detailed features & p-value & Cohen's d \\
\hline PR & 0.0001 & 1.1263 \\
QRS & 0.00004 & 1.1786 \\
QTc & 0.0323 & 0.6696 \\
pAmp & 0.0039 & 0.8821 \\
rAmp & 0.0047 & 0.8649 \\
tAmp & 0.1040 & 0.5151 \\
\hline
\end{tabular}

Table 1: P-value and effect size estimates for all features.

\section{Results and Discussion}

All calculations were done using MATLAB R2017a. Tensorlab, a toolbox for rapid prototyping of tensor decompositions, was used for all tensor calculations [7].

Table 1 presents the p-values and effect size estimates for all global and detailed features. 2 out of 4 global features and 4 out of 6 detailed features show significant differences between the stable and the unstable periods. This is also confirmed by Figure 2, which shows the boxplots for all global (Figure 2a) and detailed (Figure 2b) feaures. Analysis of Cohen's d shows that all parameters have at least a medium effect size $(d>0.5)$. The features that show significant differences between both methods all have large effect sizes $(\mathrm{d}>0.8)$, which indicate that they have a large practical significance. This can be verified on Figure 2, where the median values for these features show the most obvious contrasts.

Further inspection of Figure 2 reveals that both both features related to the QRS complex (DTW ${ }_{\mathrm{QRS}}$ and the QRS interval length) have a significantly larger range in the unstable period than the other features. This suggests that the physiological changes that lead to a cardiorespiratory arrest affect the QRS complex more seriously than the other waves. Since the QRS complex corresponds to depolarization of the ventricles, it can be an indication that ventricular detoriation plays a major role in the occurence of code blues in an ICU environment.

From Table 1, we can also conclude that the PR interval length is also an important parameter ( $p=0.0001$, Cohen's $\mathrm{d}=1.13$ ). This confirms the findings by Hu et al. [2] which also identified this parameter in a cohort of bradyasystolic patients.

While the results presented in this study are promising, the findings are still preliminary. In order to have clinical use, additional analyses about the timing and direction of these parameter changes are necessary. Furthermore we will increase the number of patients included in the study. 


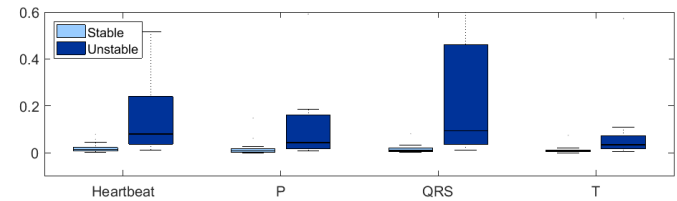

(a) Global features

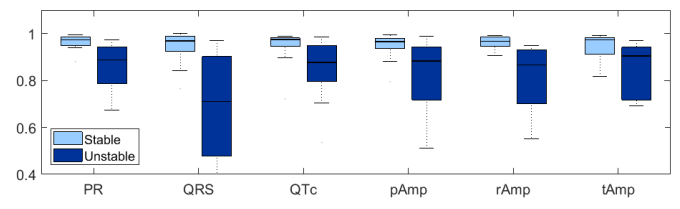

(b) Detailed features

Figure 2: Boxplots for all global (a) and detailed (b) parameters, indicating the median, 25th and 75th quantile for both periods (stable in light blue, unstable in dark blue)

Finally the type of cardiorespiratory arrest will be taken into account, since it is expected that cardiac arrests caused by ventricular fibrillation will have different characteristics than arrest caused by pulseless electrical activity.

\section{Conclusion}

In this paper we present an analysis of the changes in the ECG signal in patients prior to a in-hospital cardiorespiratory arrest. Tensors and tensor decompositions are used to represent and analyse the ECG signal. Statistical testing of 10 derived parameters (4 global and 6 detailed parameters) shows that $6 / 10$ features show significant differences between a stable period in the beginning of a recording and a unstable period directly prior to the code blue. While the presented results are preliminary, they show that tensors are a suitable way to analyse heartbeat morphology changes.

\section{Acknowledgements}

KU Leuven BOF CoE\#: PFV/10/002 (OPTEC) SPARKLE \#: IDO-13-0358 The effect of perinatal stress on the later outcome in preterm babies \#: C24/15/036 TARGID - Development of a novel diagnostic medical device to assess gastric motility \#: C32-16-00364 FWO Project \# : G.0A5513N (Deep brain stimulation) IWT Project \# : SWT 150466 - OSA+ O\& O HBC 20160184 eWatch imec funds 2017 iMinds Medical Information Technologies ICON HBC.2016.0167, 'SeizeIT' Belgian Federal Science Policy Office IUAP \# P7/19/ (DYSCO, , 2012-2017) Belgian Foreign Affairs-Development Cooperation VLIR UOS programs (2013-2019) EU: European Union's Seventh Framework Programme (FP7/20072013) EU MC ITN TRANSACT 2012, \# 316679 The HIP Trial: \# 260777 ERASMUS + INGDIVS 2016-1-SE01KA203-022114 European Research Council The research leading to these results has received funding from the European Research Council under the European Union's Seventh Framework Programme (FP7/2007-2013) / ERC Advanced Grant: BIOTENSORS (n 339804). This paper reflects only the authors' views and the Union is not liable for any use that may be made of the contained information.
This work in partially funded by R01NHLBI 128679 , R18H5022860, R01GM111378, UCSF Middle-Career scientist award, and UCSF Institute of Computational Health Sciences.

\section{References}

[1] Girotra S, Nallamothu BK, Spertus JA, Li Y, Krumholz HM, Chan PS. Trends in survival after in-hospital cardiac arrest. New England Journal of Medicine 2012; 367(20):1912-1920.

[2] Hu X, Do D, Bai Y, Boyle NG. A case-control study of nonmonitored ecg metrics preceding in-hospital bradyasystolic cardiac arrest: Implication for predictive monitor alarms. Journal of electrocardiology 2013;46(6):608-615.

[3] Do DH, Hayase J, Tiecher RD, Bai Y, Hu X, Boyle NG. Ecg changes on continuous telemetry preceding in-hospital cardiac arrests. JECG 2015;48(6):1062-1068.

[4] Goovaerts G, Vandenberk B, Willems R, Van Huffel S. Automatic detection of $\mathrm{t}$ wave alternans using tensor decompositions in multilead ecg signals. Physiological Measurement 2017;38(8):1513-1528.

[5] Padhy S, Dandapat S. Third-order tensor based analysis of multilead ecg for classification of myocardial infarction. Biomedical Signal Processing and Control 2017;31:71-78.

[6] Pan J, Tompkins WJ. A real-time qrs detection algorithm. IEEE TBME 1985;(3):230-236.

[7] Vervliet N, Debals O, Sorber L, Van Barel M, De Lathauwer L. Tensorlab user guide. wwwtensorlabnet 2016;

[8] Martínez JP, Almeida R, Olmos S, Rocha AP, Laguna P. A wavelet-based ecg delineator: evaluation on standard databases. IEEE TBME 2004;51(4):570-581.

[9] Fridericia L. The duration of systole in an ecg in normal humans and in patients with heart disease. Annals of Noninvasive Electrocardiology 2003;8(4):343-351.

[10] Sullivan G, Feinn R. Using effect size-or why the $p$ value is not enough. Technical report, JGME-D-12-00156.1.

Address for correspondence:

Griet Goovaerts

Kasteelpark Arenberg 10 - box 2446

3001 Leuven

Belgium

griet.goovaerts@esat.kuleuven.be 